\title{
THE EFFECT OF HYMENOPTERA VENOM IMMUNOTHERAPY ON NEUTROPHILS, INTERLEUKIN 8 (IL-8) AND INTERLEUKIN 17 (IL-17)
}

\section{KRZYSZTOF PAŁGAN, MAGDALENA ŻBIKOWSKA-GOTZ, ROBERT ZACNIEWSKI, and ZBIGNIEW BARTUZI}

Collegium Medicum in Bydgoszcz, Nicolaus Copernicus University, Toruń, Poland

Department of Allergology, Clinical Immunology and Internal Diseases

\begin{abstract}
Objectives: Venom immunotherapy (VIT) is an effective treatment method and is addressed to patients with a history of an anaphylactic reaction to Hymenoptera stings. However, the immunological mechanisms of protection have not been explained yet. The objective of this study was to analyze neutrophils, interleukin 8 (IL-8) and interleukin 17 (IL-17) before and after the initial phase of the immunotherapy. Material and Methods: Overall, 40 individuals, including 20 wasp venom sensitized and 20 bee venom sensitized patients, were included in the study. The patients had had a history of severe allergic reactions type III and IV according to Mueller's classification. An ultra-rush VIT protocol was used in this study. The concentration of serum IL-8 and IL-17A was determined using the ELISA enzymatic method. Results: The authors demonstrated a significant rise in the IL-8 level after the immunotherapy, compared to baseline $(14.9$ vs. $24.7, \mathrm{p}<0.05)$. The rise in the neutrophils level was also noticeable but proved to be barely out of the range of statistical significance (4.3 vs. 5.0, $\mathrm{p}=0.06)$. The shift in IL-17A was negligent and not statistically significant in the paired samples t-test $(1.6$ vs. $1.5, \mathrm{p}=0.34)$. Conclusions: Venom immunotherapy induces neutrophils and IL-8 activity after 2 days. After the desensitization, the level of IL-17A did not change. Int J Occup Med Environ Health. 2020;33(6):811-7
\end{abstract}

Key words:

bee, IL-8, IL-17A, venom immunotherapy, wasp, neutrophils

\section{INTRODUCTION}

Venom immunotherapy (VIT) is a very effective treatment in patients allergic to insect venoms. There is no doubt that VIT offers protection against future systemic reactions, prevents morbidity and mortality, and improves the quality of life. It has been estimated that VIT protects around $95 \%$ of patients allergic to Vespidae and $75-85 \%$ of those allergic to honey bees [1-3]. However, the prevention of a Hymenoptera sting severe allergic reaction has been described while the immunological mechanisms of VIT remain sketchy.
Several studies have provided evidence that VIT in the very early stages of the therapy increases both venom-specific $\operatorname{IgE}$ and $\operatorname{IgG}$ concentrations. In the long run, it gradually increases venom-specific IgG levels and induces changes in $\mathrm{T}$ cell reactivity [4]. The main mechanism of the therapy is likely due to the cytokine shift from a T helper 2 to a T helper 1 dominant pattern. The change in these immunological parameters during immunotherapy is characteristic of VIT, and this fact is reaffirmed by the decrease in interleukin 4 (IL-4) and the increase in both interferon gamma and interleukin

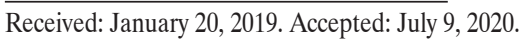

Corresponding author: Krzysztof Pałgan, Collegium Medicum in Bydgoszcz, Nicolaus Copernicus University, Department of Allergology, Clinical Immunology and Internal Diseases, Ujejskiego 75, 85-168 Bydgoszcz, Poland (e-mail: palgank@wp.pl). 
10 (IL-10) production. Another immunological observation also shows a downregulation of mast cells and basophiles reactivity [5].

Recently, the role of innate immune response, dendritic cells (DCs) and type 2 innate lymphoid cells has been discussed, along with their contribution to allergic inflammation and immunotherapy.

This work has evolved into a modern understanding of innate immunity, especially the neutrophils and cytokines associated with these cells in the early immune response during VIT, and an interface between the innate and adaptive immune responses. In this paper, the authors have examined the effect of the early phase of VIT on other immunological components like neutrophils, interleukin 17 (IL-17) and interleukin 8 (IL-8), as well as presented the results obtained with 2-day protocol immunotherapy.

\section{MATERIAL AND METHODS}

\section{Participants}

Overall, 40 individuals (14 males, 26 females) of mean age $41.03 \pm 12.43$ years were included in the study. The patients were admitted to the Department of Allergology, Clinical Immunology and Internal Diseases, Collegium Medicum in Bydgoszcz, in January 2011-December 2016. The diagnosis of Hymenoptera venom allergic reaction was confirmed with a clinical history, positive specific $\operatorname{IgE}(\mathrm{sIgE})$, and intradermal tests at the concentration of $0.001-1.0 \mu \mathrm{g} / \mathrm{ml}$ [6]. The patients had had a history of severe allergic reactions type III and IV according to Mueller's classification [7]. None of the subjects had received allergen immunotherapy for Hymenoptera venom.

\section{Wasp venom sensitized patients}

Twenty patients ( 4 men, 16 women, mean age: $41.17 \pm 9.52$ years [min.-max 18-71 years]) who had experienced systemic reactions to wasp stings were included in the study.
The time between the last sting and the hospitalization was $\mathrm{M} \pm$ SD $20.45 \pm 27.16$ months.

\section{Bee venom sensitized patients}

Twenty patients ( 10 men, 10 women, mean age: $40.9 \pm 15.35$ years [min.-max 16-68 years]) who had experienced systemic reactions to bee stings, but not to wasp stings, were included in the study. The time between the last sting and the hospitalization was $\mathrm{M} \pm \mathrm{SD} 15.39 \pm 13.53$ months.

\section{Determination of IgE}

Total and specific IgE levels were enumerated by fluoroenzyme immunoassay (ImmunoCAP FEIA) using an ImmunoCAP kit (Pharmacia, Uppsala, Sweden). Values $>100 \mathrm{kU} / \mathrm{l}$ and $0.35 \mathrm{kU} / \mathrm{l}$ for the total and specific IgE levels, respectively, were considered abnormal.

\section{VIT protocol}

An ultra-rush VIT protocol [8] was used in this study. Ten incremental doses of the venom were administered subcutaneously every $30 \mathrm{~min}$. The initial dose was $0.01 \mathrm{mg}$ of the venom, and the subsequent doses were increased until a cumulative dose of $68.5 \mu \mathrm{g}$ was administered on day 1 . The maximum dose $(100 \mu \mathrm{g})$ was reached on day 2 , and the cumulative induction dose was approximately $264.1 \mathrm{mg}$ of the venom. Venom immunotherapy was performed with the Venomenhal ${ }^{\circledR}$ (Allergen extracts - Hymenoptera venoms allergens, Hal Allergy Warsaw, Poland).

\section{Experimental procedures}

Venous blood samples for the study were taken from all subjects the day before and the day after VIT, early in the morning, after the subjects had fasted for $>12 \mathrm{~h}$ overnight.

\section{Peripheral blood neutrophils counts}

Peripheral venous blood samples were collected to ethylenediaminetera acetic acid tubes the day before and 
the day after the initial phase of VIT. An automated analyzer (Beckman Coulter, Miami, FL, USA) was used to determine the neutrophils counts.

\section{Determination of IL-17A and IL-8}

The concentrations of the serum IL- 8 and IL-17A were determined by means of the ELISA enzymatic method, using the eBioscience kit characterized by a moderate minimum detectable dose at a level of $0.5 \mathrm{pg} / \mathrm{ml}$. The test was performed according to the manufacturer's instruction. The readout and calculation of the concentrations were performed using the apparatus and software of BioTek Instruments ELx800 (BioTek Instruments, Inc., Winooski, VT, USA). All determinations were performed the day before and the day after VIT.

\section{Baseline serum tryptase}

The tryptase levels were determined by means of ImmunoCAP. Mastocytosis was excluded by examination and measurement of the serum level of tryptase. The level was in the reference range $<11.4 \mathrm{ng} / \mathrm{ml}$.

\section{Statistical analysis}

The statistical analysis was performed with SPSS Statistics version 23.0 (IBM Corp. Armonk, NY, USA). An un- paired, 2-tailed Student's t-test was performed for the statistical analysis. A p-value of $<0.05$ was required for statistical significance.

\section{Ethics statement}

The Bioethics Committee of the Nicolaus Copernicus University approved the study and each subject gave their written informed consent.

The study received the approval from the participating hospitals and all patients provided their written informed consent.

\section{RESULTS}

The authors measured the neutrophils, IL-8 and IL-17A levels before and after the patients underwent VIT. They then performed a statistical analysis using SPSS Statistics version 23.0 (IBM Corp., Armonk, NY). The rise in the neutrophils level was also noticeable, but proved to be barely out of the range of statistical significance (4.3 vs. 5.0, $\mathrm{p}=0.06$ ) (Figure 1). Using a paired samples t-test, the authors observed a statistically significant rise in the IL-8 level after the therapy, compared to baseline (14.9 vs. 24.7, p < 0.05) (Figure 2). The shift in IL-17A was negligent and not statistically significant in the paired samples t-test (1.6 vs. $1.5, \mathrm{p}=$ 0.34) (Figure 3).
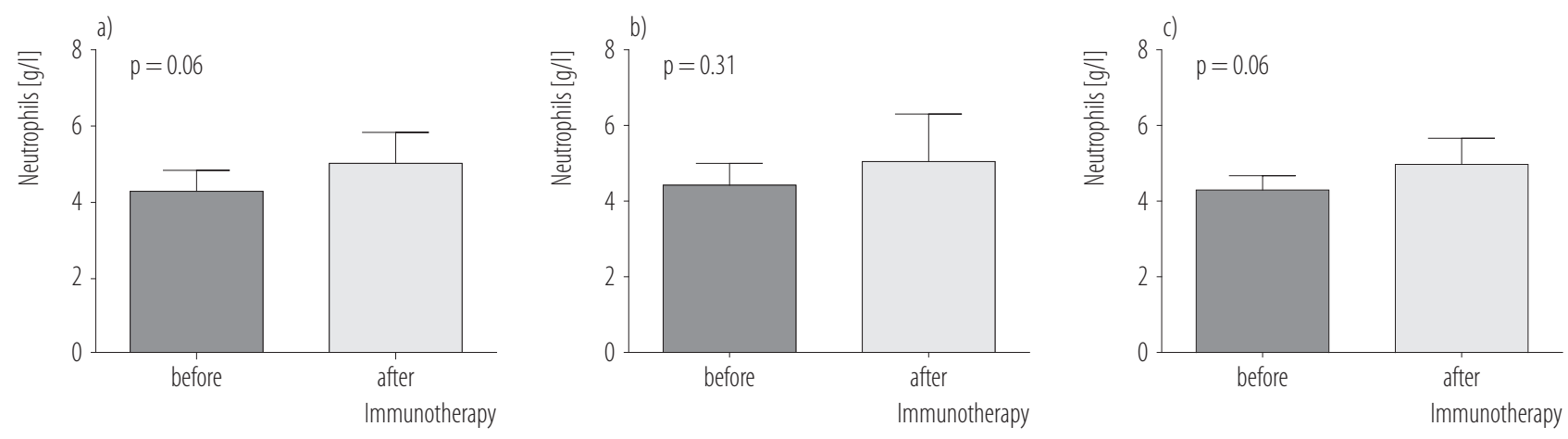

Figure 1. The effect of the initial venom immunotherapy (VIT) on peripheral blood neutrophils before and after the therapy: a) with the wasp venom, b) with the bee venom, c) with the wasp and bee venoms, in the bee and wasp venom allergic patients 

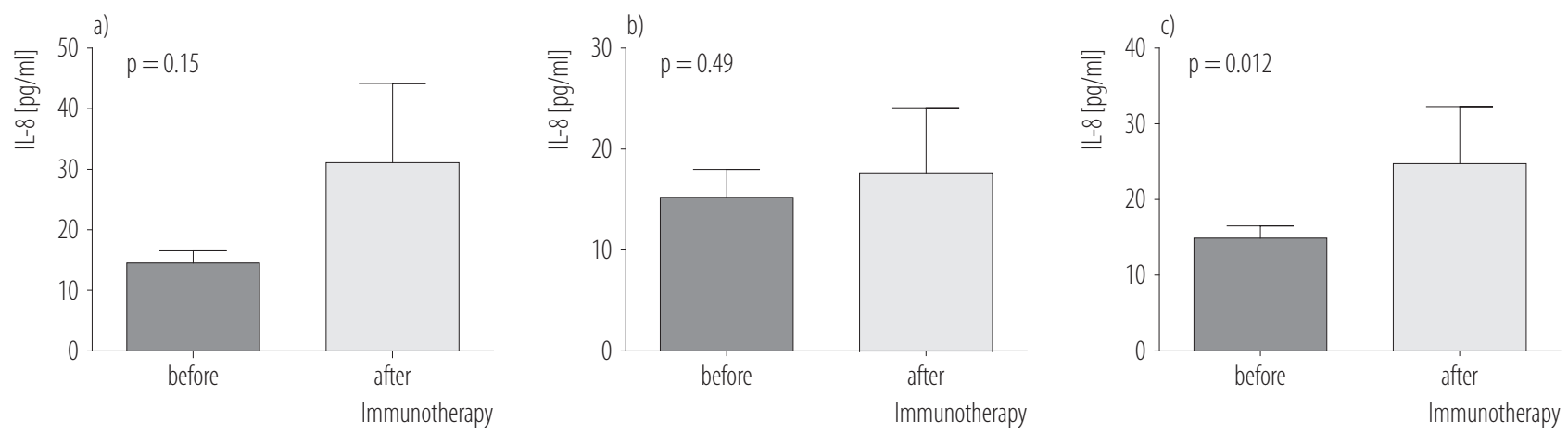

Figure 2. Interleukin 8 (IL-8) cytokine responses to immunotherapy: a) with the wasp venom, b) with the bee venom, c) with the wasp and bee venoms, in the bee and wasp venom allergic patients
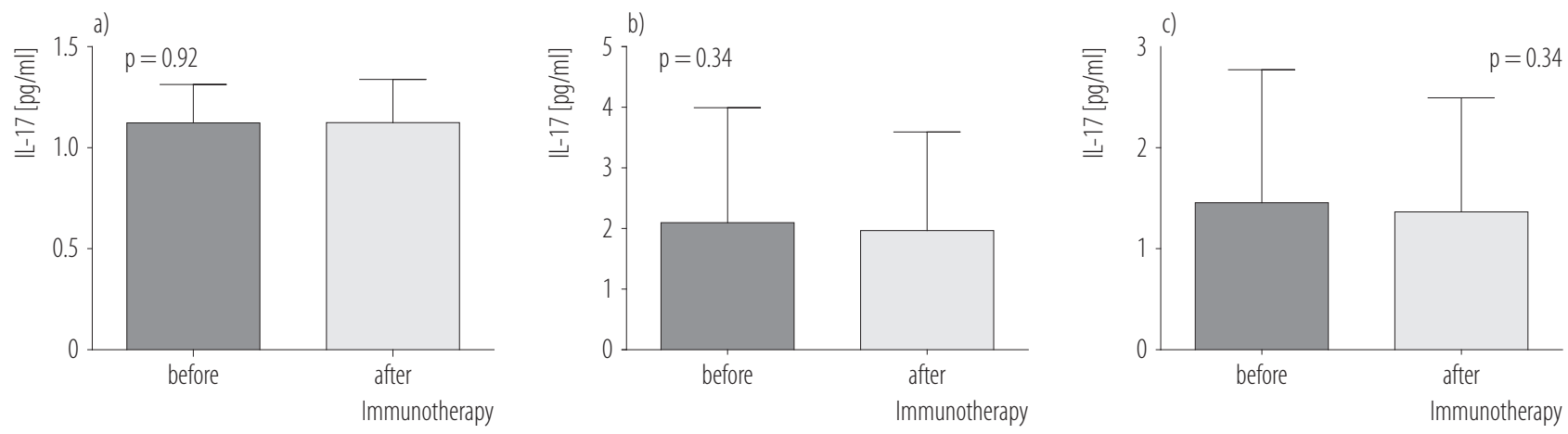

Figure 3. Interleukin 17 (IL-17) levels before and after venom immunotherapy (VIT): a) with the wasp venom, b) with the bee venom, c) with the wasp and bee venoms, in the bee and wasp venom allergic patients

In a multivariate analysis, the authors analyzed the change in outcome variables by applying an analysis of covariance with repeated measures (ANCOVA), with age, the type of the insect venom and the time since the last sting as covariates. Similar to the univariate analyses, after adjusting for the covariates, the shift in the IL-8 level proved to be statistically significant $(p<0.5)$. Moreover, the authors observed a statistically significant interaction between the type of the insect venom used in the intervention and the resulting shift in IL-8 $(\mathrm{F}=4.09, \mathrm{p}<0.05)$, as the patients treated with the wasp venom showed a more extensive change from baseline (Table 1).

During the immunotherapy, systemic reactions were not observed in all patients. Side effects were mainly of local nature, such as swelling and itching in an arm after venom injection.

\section{DISCUSSION}

Venom immunotherapy is well recognized by its efficacy, and compelling evidence implicates regulatory $\mathrm{T}$ cells (Tregs) and a significant decrease in basophiles activation in the underlying tolerogenic mechanisms [9]. Results of the study showed that, in the early stage of VIT, neutrophils and IL-8 could also have a role to play in this process. The number of neutrophils in peripheral blood samples was greater after the treatment particularly with the wasp venom $(p=0.086)$. The analysis of IL-8 concentration was also significantly greater in the wasp group $(\mathrm{p}<0.05)$. The results suggest that IL-8, as a chemoattractant for neutrophils, plays a role in the early stage of VIT. These findings are consistent with a pilot study by Davis et al. [10] on gene expression changes after rush immunotherapy. The authors were able to show that immu- 
Table 1. Neutrophils, IL-8 and IL-17 at baseline and post-initiation of venom immunotherapy (VIT)

\begin{tabular}{lcccccc}
\hline \multirow{2}{*}{ Tested parameters } & \multicolumn{2}{c}{ Neutrophils } & \multicolumn{2}{c}{$\begin{array}{c}\text { IL-8 } \\
{\left[10^{9} / 1\right]}\end{array}$} \\
\cline { 2 - 7 } & \multicolumn{2}{c}{$\mathrm{M}$} & \multicolumn{2}{c}{$\begin{array}{c}\text { IL-17 } \\
\text { [pgl } / \mathrm{ml}]\end{array}$} \\
\cline { 2 - 7 } & baseline & after VIT & baseline & after VIT & baseline & after VIT \\
\hline Bee $(\mathrm{N}=20)$ & 4.39 & 5.01 & 15.31 & 17.66 & 2.10 & 1.97 \\
Wasp $(\mathrm{N}=20)$ & 4.23 & 4.95 & 14.54 & 31.12 & 1.12 & 1.12 \\
\hline
\end{tabular}

notherapy with 4 common major aeroallergens, i.e., Bermuda grass, ragweed, and Dermatophagoides spteronyssinus and Dermatophagoides farinae, changed several gene transcripts after 1 week. Interestingly, IL-8 was strongly induced in this therapy.

Notably, IL-8 plays an important role in the rapid mobilization of hematopoietic cells during inflammatory responses [11]. Several observations have indicated that DCs play a crucial pathogenetic role in allergic diseases and allergen immunotherapy. Dreschler et al. [12] described specific changes in the number and characteristics of DCs before and after VIT. The authors observed a profound decrease in the number of blood plasmacytoid DCs (pDCs) after VIT initiation. In fact, pDCs in animal models play an anti-inflammatory role in allergic disorders [13].

Another observation made by Dreschler et al. [12] is the decrease in Toll-like receptor 2 (TLR2) expression on myeloid DCs (mDCs) which reflects a shift to a decrease in the allergic properties of mDCs after VIT. Thus, the authors hypothesized that VIT led to changes in the innate immune response and DC, and could increase IL- 8 and neutrophils activity. Furthermore, TLR led to the production of IL-6 and IL-8 in salivary glands [14,15]. A recent study has shown that neutrophils are activated in the early stage of acute human anaphylaxis [16]. Previous studies conducted by the authors of this study confirmed that neutrophils are rapidly activated during allergic food hypersensitivity and are important elements of non-specific immune response [17]. These studies suggest a potential significance of neutrophils and innate immune response in anaphylaxis and immunotherapy.

The results have prompted the authors to consider other factors participating in the early stage of immune response to VIT. Factors such as the granulocyte-macrophage colony-stimulating factor, interleukin 1 , the tumor necrosis factor or the transforming growth factor are currently being tested in the authors' clinic.

Generally, IL-17A belongs to the family of IL-17 cytokines. The group of IL-17 includes interleukins of a similar structure, marked subsequently with alphabet letters from A to F. While being mainly related to the participation of neutrophils, IL-17A is strongly a pro-inflammatory cytokine with a multidirectional mechanism of activity. The activity of IL-17A is strongly related to the cell of the innate immune response. This interleukin is capable of inducing an expression of numerous mediators of inflammatory reaction by its participation in the proliferation, maturation, migration and chemotaxy of neutrophils, which are important elements of non-specific immune response [18].

Considered an indicator of severity of allergic rhinitis, IL-17 is the cytokine released by T helper 17 lymphocytes which bridges the innate and adaptive immune responses [18]. Barczyk et al. [19] demonstrated that the serum IL-17, sputum and bronchoalveolar lavage fluid from patients with asthma positively correlated with the degree of airway hyperreactivity. There is evidence that $\mathrm{T}$ helper 17 cells can change to $\mathrm{T}$ helper 1-like cells or acquire the ability to produce IL-10 which increase during bee 
VIT [20,21]. Data obtained in this study, demonstrating the level of the serum IL-17A before and after VIT, did not reach statistical significance. The authors were unable to explain these findings. They could only speculate that the ultra-rush VIT was too short for IL-17A changes.

Differences in the wasp and bee groups might be due to different venom compositions and allergenicity. The major allergens of the honeybee (Apis mellifera) venom are phospholipase A2 (Api m 1), hyaluronidase (Api m 2) and melittin (Api m 4). The wasp (Vespula germanica, Vespula vulgaris) venom contains prominent allergens like phospholipase A1 (Ves v 1), hyaluronidase (Ves v 2.0101), hyaluronidase (Ves v 2.0201) and antigen 5 (Ves v 5). Recently, studies on phospholipase A2 have shown that the enzyme induced Treg and showed a strong capability for immunosuppressive effects. Accordingly, the authors hypothesized that Api m 2 and other components of the bee venom may have contributed to the differences in the neutrophils count and IL-8 activity, which were observed in their study.

\section{CONCLUSIONS}

It has been documented that both the innate and adaptive immune responses are modulated during allergen immunotherapy. As shown in this study, the peripheral blood number of neutrophils and the serum concentration of IL-8 increased after 2 days of VIT. The authors still know too little about the immunological mechanism of VIT. Future investigations will provide essential insights into the regulatory mechanisms of VIT and the role of innate immunity in developing resistance against these venoms.

\section{REFERENCES}

1. Ruëff F, Wenderoth A, Przybilla B. Patients still reacting to a sting challenge while receiving conventional Hymenoptera venom immunotherapy are protected by increased venom doses. J Allergy Clin Immunol. 2001;108(6):1027-32, https:// doi.org/10.1067/mai.2001.119154.
2. Golden DB. Insect sting allergy and venom immunotherapy: a model and a mystery. J Allergy Clin Immunol. 2005;115(3):439-48, https://doi.org/10.1016/j.jaci.2005.01.005.

3. Nittner-Marszalska M, Cichocka-Jarosz E, Małaczyńska T, Kraluk B, Rosiek-Biegus M, Kosinska M, et al. Safety of Ultrarush Venom Immunotherapy: Comparison Between Children and Adults. J Investig Allergol Clin Immunol. 2016; 26(1):40-7.

4. Przybilla B, Ruëff F. Hymenoptera venom allergy. J Dtsch Dermatol Ges. 2010;8(2):114-30, https://doi.org/10.1111/ j.1610-0387.2009.07125.x.

5. McHugh SM, Deighton J, Stewart AG, Lachmann PJ, Ewan PW. Bee venom immunotherapy induces a shift in cytokine responses from a TH-2 to a TH-1 dominant pattern: comparison of rush and conventional immunotherapy. Clin Exp Allergy. 1995;25:828-38, https://doi.org/10.1111/ j.1365-2222.1995.tb00025.x.

6. Cichocka-Jarosz E, Stobiecki M, Brzyski P, Rogatko I, Nittner-Marszalska M, Sztefko K, et al. Simplification of intradermal skin testing in Hymenoptera venom allergic children. Ann Allergy Asthma Immunol. 2017;118(3):32632, https://doi.org/10.1016/j.anai.2016.11.006.

7. Mueller UR. Insect sting allergy. Clinical picture, diagnosis and treatment. Stuttgart: Gustav Fischer; 1990. p. 35-46.

8. Nittner-Marszalska M, Cichocka-Jarosz E. Insect sting allergy in adults: key messages for clinicians. Pol Arch Med Wewn. 2015;125:929-37.

9. Trabado RA, Hijón CC, Cantariño RA, Romero-Chala S, García-Trujillo JA, Pereira FLM. Short-, Intermediate-, and Long-Term Changes in Basophil Reactivity Induced by Venom Immunotherapy. Allergy Asthma Immunol Res. 201;8(5):412-20, https://doi.org/10.4168/aair.2016.8.5.412.

10. Davis LS, Bhutani S, Barnett SR, Khan DA. Early gene expression changes with rush immunotherapy. Clin $\mathrm{Mol} \mathrm{Al-}$ lergy. 2011;9:12, https://doi.org/10.1186/1476-7961-9-12.

11. Kehrl JH. Chemoattractant receptor signaling and the control of lymphocyte migration. Immunol Res. 2006;34:211-27, https://doi.org/10.1385/IR:34:3:211. 
12. Dreschler K, Bratke K, Petermann S, Bier A, Thamm P, Kuepper M, et al. Impact of immunotherapy on blood dendritic cells in patients with Hymenoptera venom allergy. J Allergy Clin Immunol. 2011;127(2):487-94.e4943, https:// doi.org/10.1016/j.jaci.2010.12.003.

13. Kool M, van Nimwegen M, Willart MA, Boon L, Smit JJ, Coyle A, et al. An anti-inflammatory role for plasmacytoid dendritic cells in allergic airway inflammation. J Immunol. 2009;183(2):1074-82, https://doi.org/10.4049/jimmunol.09 00471.

14. Ohta K, Ishida Y, Fukui A, Mizuta K, Nishi H, Takechi MT, et al. Toll-like receptor (TLR) expression and TLR-mediated interleukin-8 production by human submandibular gland epithelial cells. Mol Med Rep. 2014;10(5):2377-82, https:// doi.org/10.3892/mmr.2014.2507.

15. Pałgan K, Bartuzi Z, Zbikowska-Götz M. Dendritic cells and allergy. Pneumonol Alergol Pol. 2009;77(6):528-32.

16. Francis A, Bosio E, Stone SF, Fatovich DM, Arendts G, Nagree $\mathrm{Y}$ et al. Neutrophil activation during acute human anaphylaxis: analysis of MPO and sCD62L. Clin Exp Allergy. 2017;47(3):361-70, https://doi.org/10.1111/cea.12868.
17. Żbikowska-Gotz M, Pałgan K, Gawrońska-Ukleja E, Kuźmiński A, Przybyszewski M, Socha E, et al. Expression of IL-17A concentration and effector functions of peripheral blood neutrophils in food allergy hypersensitivity patients. Int J Immunopathol Pharmacol. 2016;29(1):90-8, https://doi. org/10.1177/0394632015617069.

18. Qiu Q, Lu H, Lu C, Chen S, Han H. Variations in TGF-beta, IL-10, and IL-17 after specific immunotherapy and correlations with symptoms in patients with allergic rhinitis. J Investig Allergol Clin Immunol. 2012;22(4):311-2.

19. Barczyk A, Pierzchala W, Sozanska E. Interleukin-17 in sputum correlates with airway hyperresponsiveness to methacholine. Respir Med. 2003;97:726-33.

20. Ferretti E, Ponzoni M, Doglioni C, Pistoia V. IL-17 superfamily cytokines modulate normal germinal center B cell migration. J Leukoc Biol. 2016;100(5):913-8.

21. Akdis CA, Blesken T, Akdis M, Wüthrich B, Blaser K. Role of interleukin 10 in specific immunotherapy. J Clin Investig. 1998;102(1):98-106.

This work is available in Open Access model and licensed under a Creative Commons Attribution-NonCommercial 3.0 Poland License - http://creativecommons.org/ licenses/by-nc/3.0/pl/deed.en. 